TRANSACTIONS OF THE

AMERICAN MATHEMATICAL SOCIETY

Volume 357, Number 3, Pages 983-998

S 0002-9947(04)03762-6

Article electronically published on October 19, 2004

\title{
ON THE CONSTRUCTION OF CERTAIN 6-DIMENSIONAL SYMPLECTIC MANIFOLDS WITH HAMILTONIAN CIRCLE ACTIONS
}

\author{
HUI LI
}

\begin{abstract}
Let $(M, \omega)$ be a connected, compact 6-dimensional symplectic manifold equipped with a semi-free Hamiltonian $S^{1}$ action such that the fixed point set consists of isolated points or surfaces. Assume $\operatorname{dim} H^{2}(M)<3$. In an earlier paper, we defined a certain invariant of such spaces which consists of fixed point data and twist type, and we divided the possible values of these invariants into six "types". In this paper, we construct such manifolds with these "types". As a consequence, we have a precise list of the values of these invariants.
\end{abstract}

\section{INTRODUCTION}

Assume that $(M, \omega)$ is a connected, compact 6-dimensional symplectic manifold equipped with a semi-free (free outside fixed point set) Hamiltonian $S^{1}$ action such that the fixed point set consists of isolated points or surfaces. For the case dim $H^{2}(M)<3$, in $[\mathrm{L}$, we defined a certain invariant which consists of "fixed point data" and "twist type", and we gave a complete list of the possible values of these invariants. We have seen that only six "types" can occur (see Theorem 1 below). We showed the existences for a few cases by giving explicit examples, such as coadjoint orbits or toric varieties. In this paper, we illustrate a method for constructing such manifolds. As a consequence, this proves the existence of the manifolds with those prescribed types as in $[\mathrm{L}]$. The techniques we use in this paper are quite different from those we used in $[\mathrm{L}$. We mainly use symplectic topological method and related results on symplectic 4 -manifolds.

Recall that the moment map $\phi$ is a perfect Morse-Bott function. The fixed point sets of the circle action are exactly the critical sets of $\phi$. Each critical set has even index. $\phi$ has a unique local minimum and a unique local maximum ([A] $)$. Recall the following definitions from $[\mathrm{L}]$

Definition 1. Let $(M, \omega)$ be a connected, compact 6-dimensional symplectic manifold equipped with a semi-free Hamiltonian $S^{1}$ action such that the fixed point set consists of isolated points or surfaces. Assume $\operatorname{dim} H^{2}(M)<3$. For each such

Received by the editors May 5, 2003.

2000 Mathematics Subject Classification. Primary 53D05, 53D20; Secondary 57R17.

Key words and phrases. Symplectic manifold, Hamiltonian $S^{1}$ action, moment map, symplectic quotient, symplectic submanifolds.

The author acknowledges the support of the center of Analysis, Geometry, and Dynamical systems in Lisbon, Portugal, where this paper was revised.

(C)2004 American Mathematical Society 
manifold, we define the fixed point data to be the diffeomorphism type of the fixed point sets, their indices, and $b_{\text {min }}$, where $b_{\text {min }}$ denotes the 1st Chern number of the normal bundle of the minimum when the minimum is a surface.

If the minimum (or the maximum) is a surface, then for a regular value right above the minimum (or right below the maximum), the reduced space is diffeomorphic to the total space of an $S^{2}$ fibration over the minimum (or over the maximum). When the fixed point set consists only of surfaces, the reduced spaces at the nonextremal values are all diffeomorphic, and there is a canonical isomorphism between their (co)homology groups (see [GS]).

Definition 2. Let $(M, \omega)$ be a compact connected 6-dimensional symplectic manifold equipped with a semi-free Hamiltonian circle action. Assume the fixed point set consists only of surfaces. If the fibers of the reduced spaces at a regular level right above the minimum and right below the maximum can be represented by the same cohomology class of the reduced space, we say there is notwist; otherwise, we say there is a twist.

In $[\mathrm{L}$, we have shown the following

Theorem 1. Let $(M, \omega)$ be a connected compact 6 -dimensional symplectic manifold equipped with a semi-free Hamiltonian circle action such that the fixed point set consists of isolated points or surfaces. Assume $\operatorname{dim} H^{2}(M)<3$. Then the possible fixed point data and the twist type are as shown in Figure 1, where $g, g_{1}$ are the genuses of the surfaces, and $b_{\max }$ is the 1 st Chern number of the normal bundle of the maximum.

Remark 1. Figure 1 gives us an order of how the moment map crosses the fixed point sets. For instance, a manifold of type (1) means that the manifold has an isolated minimum, an index 2 sphere, and an isolated maximum.

For a manifold of type (2), the two index 2 spheres cannot be on the same level of the moment map.

For manifolds of type (3) and (5), the moment map can cross the two nonextremal fixed point sets in any order or the 2 fixed point sets are on the same level of the moment map.

Remark 2. In $[\mathrm{L}$, we have seen that when there is an index 2 surface or the maximum is a surface in a certain "type", the 1st Chern numbers of the normal bundles of these surfaces are uniquely determined by the fixed point data and the twist type. (This is the reason that $b_{\max }$ is not included in the definition of the fixed point data.) For instance, for manifolds of type (1), the 1st Chern numbers of the positive normal bundle and the negative normal bundle of the index 2 sphere are both 2 ; for manifolds of type (2), the 1st Chern numbers of the positive normal bundle and the negative normal bundle of the 1st (counting from bottom) index 2 sphere are 0 and 1, respectively, and those of the second index 2 sphere are respectively 1 and 0 . Notice that in type (4) and type (5), $b_{\min }$ and $b_{\max }$ have fixed values, in types (3), (6a) and (6b), on the other hand, $b_{\min }$ can vary, and in particular, for type (6a), the genuses $g$ and $g_{1}$ can vary. Different values of these "variables" of course give different manifolds.

Remark 3. We have shown in $\mathrm{L}$ that there exists a manifold of type (1) which is a coadjoint orbit of $S O(5)$. We constructed toric varieties for type (3) when $b_{\min }=1$ 


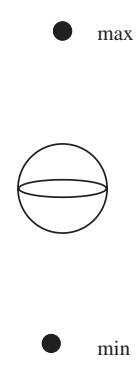

(1)
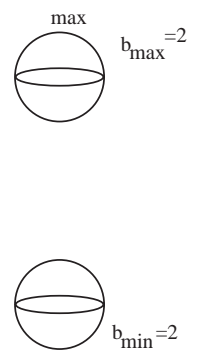

$\min$

(4) twist

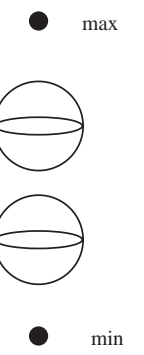

(2)

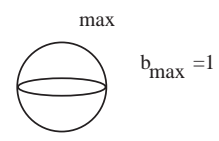

index $2 \bigcirc$ index 4

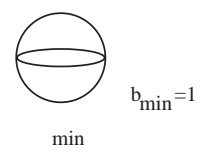

(5)
- $\max$
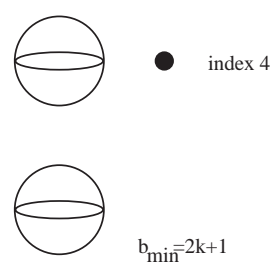

$\min$

(3)

(or this one upside down)

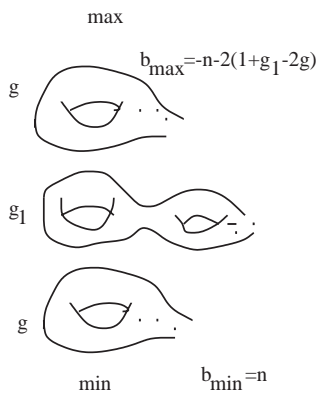

(6a) no twist

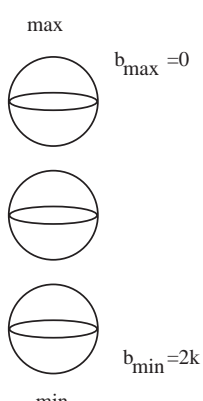

(6b) twist

(or this one upside down)

Figure 1.

and $b_{\min }=3$, and for type (4) and for type $(6 \mathrm{~b})$ when $b_{\min }=2$. Moreover, we have shown that all manifolds of type (4) are diffeomorphic to $\mathbb{C} P^{3}$, and all manifolds of type $(6 \mathrm{~b})$ with $b_{\text {min }}=2$ are diffeomorphic to $\mathbb{C} P^{3}$ blow up at a point.

In [L] and in this paper, the fixed point data has a certain meaning, for the sake of convenience. More generally, similar data (looser or stronger) is an important tool for the study of symplectic Lie group actions, especially for the study of Hamiltonian Lie group actions. It captures important geometrical and topological information about the manifolds. As we have seen in [L], for a fixed "type" in Theorem 1 , the fixed point data uniquely determines the equivariant (ordinary) cohomology ring and equivariant (ordinary) Chern classes of the manifold. More significantly, for some cases, this data classifies the manifolds up to diffeomorphism (see $[\mathbb{L}]$ ). Recall that for all the types of manifolds in Theorem 1, the reduced spaces (except at the extremal values) are $\mathbb{C} P^{2}$ or ruled manifolds. We well understand the symplectic structures of these 4-manifolds and their symplectic submanifolds. In this paper, we use this special feature to construct manifolds with the types prescribed in Theorem 1. We will show that for each "type" in Theorem 1, except for type (6a) with $1+g_{1}-2 g \leq 0$, this "local" data (related in a global way, however) allows us to construct a "global" manifold with the prescribed data. In fact, except for type (6a) with $1+g_{1}-2 g \leq 0$, this data uniquely determines the inverse image (under the moment map) of a small neighborhood of each critical or regular value 
of the moment map up to equivariant symplectomorphism (see Section 2). Let us call these "local pieces". When we construct these "local pieces", the prescribed type only gives us the cohomology classes of the reduced "symplectic forms". We need to make sure that these cohomology classes do contain symplectic forms. A "local piece" for the minimum or the maximum can always be constructed. The main problem is to construct a "local piece" for an index 2 surface. In this case, we need 4 pieces of information $\left(M_{0}, \omega_{0}, P, X\right)$ (see Section 2 for its meaning), where $M_{0}$ is the reduced space at this critical level, and $X$ is a symplectic submanifold of $M_{0}$. More precisely, $X$ is the symplectically embedded image of the index 2 surface in $M_{0}$ (see Section 2). The prescribed type must make this to be true. For (6a), if $1+g_{1}-2 g \leq 0$, the dual class (which can be computed from the "type") of the surface in the reduced space cannot represent the symplectically embedded image of the index 2 surface. This is the reason we failed to construct manifolds for type (6a) when $1+g_{1}-2 g \leq 0$. In order to construct the manifolds globally, we need to perform some gluing along some regular levels. The main reason we can perform the gluing is that for a regular level of the moment map, two different symplectic forms in the same cohomology class of the reduced space (which is $\mathbb{C} P^{2}$ or a ruled manifold) are diffeomorphic. These classification results on the symplectic structures on $\mathbb{C} P^{2}$ or ruled manifolds are due to Taubes and Lalonde-McDuff ([T], $[\overline{\mathrm{LMc}}])$.

In [L] and in this paper, we assume that $\operatorname{dim} H^{2}(M)<3$. Under this assumption, we define fixed point data. As we mentioned in Remark 2, when there is an index 2 surface or the maximum is a surface in a certain "type", the 1st Chern numbers of the normal bundles of these surfaces are uniquely determined by the fixed point data. Notice that the definition of twist does not depend on this restriction. However, it is defined under the assumption that the fixed point set consists only of surfaces. If we remove the assumption $\operatorname{dim} H^{2}(M)<3$, we have more fixed point set components. For the definition of fixed point data, we need to specify more 1st Chern numbers of the normal bundles of the fixed point set components to determine those of the other fixed point set components. Alternatively, depending on the purposes, we may assume that all these Chern numbers for all the fixed point set components are compatibly given and we include all of them in the definition. If the fixed point set consists only of surfaces, we can still have the same definition of twist. If the minimum and the maximum are surfaces (with the same genus, see $[\mathrm{L}$ ), and the other fixed point set consists of surfaces, index 2 or index 4 isolated points, then the reduced spaces change not only by diffeomorphism, but also by blow ups or blow downs (GS). The reduced spaces and their cohomology rings are more complicated. The definition of twist needs to be properly addressed. For the construction of these more complicated manifolds, we may not understand the symplectic structures on the reduced spaces well enough to do the local construction or to perform the global gluing. Certainly, in good cases, if our requrements are satisfied, we can still construct the manifolds with certain "types" other than those in Theorem 1.

In summary, in order to construct a global manifold from a prescribed type, we need the "data" to be "compatibly matched", and we need the reduced spaces to be "good enough" to allow us to perform the gluing. The idea itself can be applied to more general constructions of Hamiltonian $S^{1}$ manifolds, for instance, for nonsemi-free Hamiltonian circle actions on other dimensional symplectic manifolds with 
other fixed point set components. Or more generally, depending on what is given, the idea itself may be applied to the setting with no Hamiltonian circle actions.

The main result of this paper is

Theorem 2. There exists a manifold for any of the prescribed fixed point data and twist type as in Theorem 1, except that for type (6a), the genuses $g$ and $g_{1}$ must satisfy $1+g_{1}-2 g>0$.

Recall that for type (6a), we computed the dual class $\eta$ of the index 2 surface in the reduced space at the critical level in $\left[\mathrm{L}: \eta=\left(1+g_{1}-2 g\right) x+2 y\right.$ for even $n$, and $\eta=\left(2+g_{1}-2 g\right) x+2 y$ for odd $n$ (see Section 3 for the meaning of $x$ and $y$ ). If $1+g_{1}-2 g \leq 0$, then these dual classes cannot represent the connected symplectically embedded index 2 surface in the reduced spaces. The class $2 y$ (for even $n$ ) or $x+2 y$ (for odd $n$ ) represents non-connected symplectic submanifolds. The reason that the classes $\eta=\left(1+g_{1}-2 g\right) x+2 y$ or $\eta=\left(2+g_{1}-2 g\right) x+2 y$ with $1+g_{1}-2 g<0$ cannot represent the symplectically embedded image of the genus $g_{1}$ index 2 surface is: the projection of the embedded surface (represented by $\eta=\left(1+g_{1}-2 g\right) x+2 y$ or $\left.\eta=\left(2+g_{1}-2 g\right) x+2 y\right)$ to the "base" of the $S^{2}$ fibration (represented by $y$ ) is a smooth degree 2 map from a genus $g_{1}$ Riemann surface to a genus $g$ Riemann surface. By the analogue Hurwitz formula for smooth maps between Riemann surfaces (see Theorem 1 in $\left[\mathrm{HY}\right.$ ), $g_{1}$ and $g$ must satisfy $1+g_{1}-2 g \geq 0$. This is the reason we cannot construct such manifolds. This fact also helps us to strengthen Theorem [1]

Corollary 1. For type (6a) in Theorem 1 the genuses of the surfaces must satisfy $1+g_{1}-2 g>0$.

Theorem 1 and this corollary give us a complete list of the possible "types". Theorem 2 shows that there exist manifolds of all these "types". Together, they give us exactly the "types" of manifolds that can occur under the assumptions of Theorem 1

\section{Construction of neighborhoods \\ OF CRITICAL AND REGULAR LEVEL SETS OF THE MOMENT MAP}

Construction of a neighborhood of the minimum:

If the minimum is isolated, by the equivariant Darboux-Weinstein theorem, a neighborhood of the minimum is equivariantly symplectomorphic to $\mathbb{C}^{3}$ with the standard symplectic form and $S^{1}$ action with weights $1,1,1$.

If the minimum is a surface $\Sigma_{g}$, then a neighborhood of the minimum is a $\mathbb{C}^{2}$ bundle $E$ over $\Sigma_{g}$. It is easy to construct such a bundle with 1 st Chern number $b_{\min }$. (We can always construct 2 line bundles with Euler numbers $m$ and $n$ for any integer $m$ and $n$ and take the sum of the line bundles.) We can put a symplectic form on a neighborhood of the zero section of $E$ (see [GS]) and make $S^{1}$ act semi-freely by fixing the base and rotating the fibers. More precisely, let $F$ be the unitary frame bundle of $E$ (assume there is a Hermitian metric on $E$ ), let $T^{*} F$ be the cotangent bundle and let $\omega_{F}$ be the canonical symplectic form on $T^{*} F$. A connection on $F$ can give us the vertical cotangent bundle $V^{*} F$. Let $i: V^{*} F \hookrightarrow T^{*} F$ be the inclusion, and let $\omega_{B}$ be the symplectic form on the base $\Sigma_{g}$. Then $\omega_{V}=i^{*} \omega_{F}+\pi^{*} \omega_{B}$ is a

\footnotetext{
${ }^{1}$ The construction procedure gave me a good reason to study the symplectic submanifolds of the reduced spaces. This helped me to discover this piece of information.
} 
symplectic form on $V^{*} F$. The $U(2)$ action on $V^{*} F$ is Hamiltonian, so is the $U(2)$ action on $\mathbb{C}^{2}$. Take $V^{*} F^{-} \times \mathbb{C}^{2}$ with the product symplectic structure, where $V^{*} F^{-}$ is $V^{*} F$ with the form $-\omega_{V}$. There is a Hamiltonian $S^{1}$ action on this space: it acts trivially on the first factor and it acts on the second factor by rotation with weights 1,1 . We can get a neighborhood of the zero section of $E$ with a symplectic form by reducing the $U(2)$ action at 0 . The semi-free Hamiltonian $S^{1}$ action remains on $E$. By the equivariant Darboux-Weinstein theorem, any $S^{1}$-invariant symplectic structure on the above neighborhood is equivariantly isomorphic to the above one.

Notice that the above construction works for other dimensions and for non-semifree $S^{1}$ actions.

Hence, we have the following lemma for the construction of a neighborhood of the minimum.

Lemma 1. Assume $F$ is an isolated point or is a closed surface. Then we can construct uniquely up to isomorphism $a(n)$ (open) Hamiltonian $S^{1}$ symplectic manifold $M_{\left[\text {min, }_{0}\right)}$ such that $F$ is the minimum of the moment map, and such that $F$ has any prescribed 1 st Chern number of its normal bundle in $M_{\left[\min , \epsilon_{0}\right)}$ when $F$ is a surface.

To construct a neighborhood of a critical level which contains an index 2 point or an index 2 surface, we can use the result of Guillemin and Sternberg ([GS]):

Assume we have $\left(M_{0}, \omega_{0}, P, X\right)$, where $M_{0}$ is a compact symplectic manifold with symplectic form $\omega_{0}, X$ is a symplectic submanifold of $M_{0}$, and $P$ is a principal $S^{1}$ bundle over $M_{0}, \pi: P \rightarrow M_{0}$. Given a small open interval $I=(-\epsilon,+\epsilon)$, we can construct a symplectic manifold $M_{I}$ with a semi-free Hamiltonian $S^{1}$ action with proper moment map $\phi$ which maps $M_{I}$ onto $I$. The symplectic form on $M_{I}$ is determined by $\omega_{0}$, the normal bundle of $X$ in $M_{0}$ and the Euler class of the circle bundle $P$ over $M_{0}$. Moreover, 0 is an index 2 critical level of $\phi$. For $-\tau<0$, $\phi^{-1}(-\tau)$ is diffeomorphic as an $S^{1}$ space to $P$, the reduced spaces of $M_{I}$ at $-\tau$ and 0 are diffeomorphic to $M_{0}$, and the reduced space of $M_{I}$ at $\tau>0$ is the blow up of $M_{0}$ along $X$. The reduced symplectic forms satisfy the Duistermaat-Heckman formula $([\mathrm{DH}])$. Moreover, $M_{I}$ is unique up to $S^{1}$ - equivariant symplectomorphisms (Theorem 13.1 in GS]).

We summarize this in

Lemma 2. Assume we have $\left(M_{0}, \omega_{0}, P, X\right)$, where $M_{0}$ is a compact symplectic manifold with symplectic form $\omega_{0}, X$ is a symplectic submanifold of $M_{0}$, and $P$ is a principal $S^{1}$ bundle over $M_{0}, \pi: P \rightarrow M_{0}$. Given a small open interval $I=(-\epsilon,+\epsilon)$, we can construct uniquely up to isomorphism a semi-free Hamiltonian $S^{1}$ symplectic manifold $M_{I}$ with moment map $\phi$ (which maps $M_{I}$ onto $I$ ) such that $\phi^{-1}(0)$ is a critical level with an index 2 fixed point set which is diffeomorphic to $X, \phi^{-1}(-\tau)$ is diffeomorphic as an $S^{1}$ space to $P$, and the reduced spaces of $M_{I}$ at $-\tau$ and 0 are diffeomorphic to $M_{0}$, the reduced space of $M_{I}$ at $\tau>0$ is the blow up of $M_{0}$ along $X$.

We can similarly construct a neighborhood of a critical level set which contains an index 4 fixed point set component by looking at it upside down.

The construction of a neighborhood of the maximum is similar to the construction of a neighborhood of the minimum.

The above construction of neighborhoods of critical levels can only be done for small intervals of critical values of $\phi$. When we construct our manifolds, we may 
need a "longer" neighborhood of a regular level. For this purpose, we construct a "regular neighborhood".

If $I$ is a connected open interval consisting of regular values of $\phi$, then $\phi^{-1}(I)$ is $S^{1}$-equivariantly diffeomorphic to $\phi^{-1}(a) \times I$, where $a \in I$. The $S^{1}$-invariant symplectic form on $\phi^{-1}(I)$ is determined by the symplectic forms on the reduced spaces and the Euler class of the principal circle bundle $\phi^{-1}(a)$ over $M_{a}$.

Lemma 3 ([Mc1]). For an interval I consisting of regular values of $\phi$, if $\omega$ is the symplectic form on $\phi^{-1}(I)$, and $\tau_{s}$ is the reduced symplectic form at $s \in I$, then $\omega$ is determined by the forms $\tau_{s}$ up to an $S^{1}$ equivariant diffeomorphism which preserves the level sets of $\phi$.

See Mc1 for details.

Assume that these neighborhoods we constructed above are "big enough" so that we can shrink them a little such that they are manifolds with boundaries, the boundaries being regular level sets of $\phi$. We will call these neighborhoods with boundaries of critical levels and regular levels "local pieces".

\section{Problems for local and global COnstructions}

We have seen in Section 2 what we need to prescribe to do the local construction. Let us review in this section what information the "types" in Theorem 1 can give us and what we need to take care of when we do the local and global constructions.

Assume $a$ is a regular value of the moment map $\phi$. Then $S^{1}$ acts freely on $\phi^{-1}(a)$, so we have a principal circle bundle

$$
\begin{aligned}
S^{1} \hookrightarrow & \phi^{-1}(a)=P_{a} \\
& \downarrow \\
& \phi^{-1}(a) / S^{1}=M_{a},
\end{aligned}
$$

where $M_{a}$ is the reduced space at $a$.

Assume the minimum (or the maximum) of the moment map is a surface $\Sigma_{g}$. Let $a$ be a regular value right above the minimal value (or right below the maximal value) such that there are no other critical sets in the neighborhood except the minimum (or the maximum). Then $M_{a}$ is the total space of a sphere bundle over $\Sigma_{g}$. Let $S^{2} \times \Sigma_{g}$ be the trivial bundle, and let $E_{\Sigma_{g}}$ be the non-trivial bundle. Let $e\left(P_{a}\right)$ be the Euler class of the circle bundle $P_{a}$ over $M_{a}$.

For $S^{2} \times \Sigma_{g}$, let $x, y \in H^{2}\left(S^{2} \times \Sigma_{g}\right)$ be a basis which consists of the dual classes of the fiber $S^{2}$ and the base $\Sigma_{g}$, respectively. Then $\int_{b a s e} x=\int_{S^{2} \times \Sigma_{q}} x y=$ $1, \quad \int_{\text {fiber }} y=\int_{S^{2} \times \Sigma_{g}} y x=1, \quad \int_{\text {fiber }} x=\int_{S^{2} \times \Sigma_{g}} x^{2}=0$, and $\int_{b a s e} y=\int_{S^{2} \times \Sigma_{g}} y^{2}=$ 0 .

Similarly, for the non-trivial bundle $E_{\Sigma_{g}}$, let $x, y \in H^{2}\left(E_{\Sigma_{g}}\right)$ be a basis which consists of the dual classes of the fiber and the section $\Sigma_{-}$which has self-intersection -1 , respectively. Then $\int_{\Sigma_{-}} x=\int_{E_{\Sigma_{g}}} x y=1, \quad \int_{\text {fiber }} y=\int_{E_{\Sigma_{g}}} x y=1, \quad \int_{\text {fiber }} x=$ $\int_{E_{\Sigma_{g}}} x^{2}=0$, and $\int_{\Sigma_{-}} y=\int_{E_{\Sigma_{g}}} y^{2}=-1$.

We have the following lemmas

Lemma $4\left([\overline{\mathrm{L}})\right.$. If the minimum is a surface $\Sigma_{g}$ with 1 st Chern number $b_{\text {min }}$ of its normal bundle, then $M_{a}$ (a above the minimum) is diffeomorphic to $S^{2} \times \Sigma_{g}$ if and only if $b_{\min }=2 k$ is even, and it is diffeomorphic to $E_{\Sigma_{g}}$ if and only if $b_{\min }=2 k+1$ is odd. In either case, $e\left(P_{a}\right)=k x-y$. 
Lemma $5([\mathrm{~L}])$. If the maximum is a surface $\Sigma_{g}$ with 1 st Chern number $b_{\max }$ of its normal bundle, then $M_{a}$ (a below the maximum) is diffeomorphic to $S^{2} \times \Sigma_{g}$ if and only if $b_{\max }=2 k^{\prime}$ is even, and it is diffeomorphic to $E_{\Sigma_{g}}$ if and only if $b_{\max }=2 k^{\prime}+1$ is odd. In either case, $e\left(P_{a}\right)=-k^{\prime} x+y$.

Let $c$ be a critical value of the moment map $\phi$, and let $S \subset \phi^{-1}(c)$ be an index 2 fixed surface. Assume for small $\epsilon, S$ is the only critical set in $\phi^{-1}([c-\epsilon, c+\epsilon])$. Let $P_{c-\epsilon}$ be the circle bundle $\phi^{-1}(c-\epsilon)$ over $M_{c-\epsilon}$, and let $e\left(P_{c-\epsilon}\right)$ be its Euler class. $P_{c+\epsilon}$ and $e\left(P_{c+\epsilon}\right)$ have similar meanings. By GS or Mc1, $M_{c+\epsilon}$ is diffeomorphic to $M_{c-\epsilon}$ (in dimension 6); let us call them $M_{r e d}$ (reduced space). Moreover, there is a symplectic embedding $i: S \rightarrow M_{\text {red }}$ such that if $i(S)=Z$, and $\eta$ is the dual class of $Z$ in $M_{\text {red }}$, then

$$
e\left(P_{c+\epsilon}\right)=e\left(P_{c-\epsilon}\right)+\eta .
$$

By [GS, when the moment map crosses an index 2 point (index 4 point), the reduced space changes by a blow up at a point (blow down of an exceptional divisor). Now let $M_{-}$be the reduced space before surgery (blow up or blow down), let $M_{+}$be the reduced space after surgery, let $e\left(P_{-}\right)$be the Euler class of the principal circle bundle over $M_{-}$, and let $e\left(P_{+}\right)$be the Euler class of the principal circle bundle over $M_{+}$. Let $\eta_{e x, \text { div }}$ be the dual class of the exceptional sphere in $M_{+}$after blow up or in $M_{-}$before blow down. Let us always call the blow down map by $\beta$. By GS, we have the following:

Corresponding to blow up,

$$
e\left(P_{+}\right)=\beta^{*} e\left(P_{-}\right)+\eta_{e x, d i v} .
$$

Corresponding to blow down,

$$
\beta^{*} e\left(P_{+}\right)=e\left(P_{-}\right)+\eta_{e x, d i v} .
$$

For each type of manifold in Theorem 1, we can use Lemma 4, Lemma 5 and (2), (3), (4) to compute the Euler classes of the principal $S^{1}$ bundles (1) for regular levels in terms of the fixed point data. By Duistermaat-Heckman formula ( $[\mathrm{DH})$, we can compute the cohomology classes of the reduced symplectic forms on the reduced spaces.

Recall that for the types of manifolds in Theorem 1 the reduced spaces are all smooth manifolds (see Section 10 in [GS]). Moreover, from above we see that (except at the minimal value and at the maximal value) they are diffeomorphic to either $\mathbb{C} P^{2}$ or the total space of an $S^{2}$ bundle over a Riemann surface (assume for manifold of type (5) that the moment map crosses the index 4 point before it crosses the index 2 point). Now, let us list the problems we should take care of for the construction:

1. The cohomology classes of the reduced forms given by Duistermaat-Heckman formula are in terms of the fixed point data, the size of the minimum (maximum) if the minimum (maximum) is a surface, and the distances between the moment map images of the fixed point set components. The question is, which cohomology class in the reduced space contains a symplectic form.

2. If $F$ is an index 2 fixed surface, then $F$ is symplectically embedded in the reduced space at the critical level (and nearby regular levels). As we have seen (see [L] for details), in each case, the dual cohomology class of $F$ in the reduced space can be computed in terms of the fixed point data. The question is, which dual class can be represented by a symplectic submanifold. 
3. When we glue the "local pieces" together, we glue them along a regular level. Since this regular level is a principal $S^{1}$ bundle over the reduced space, we can glue the two reduced spaces on the local pieces together (the fibers will be identified correspondingly). We only know that the cohomology classes of the reduced symplectic form are the same on these two reduced spaces; the symplectic forms may be chosen differently. The question is, if there is a diffeomorphism between the two reduced spaces such that one form is pulled to another by this diffeomorphism.

Section 4 and Section 5 will give answers to the above questions.

\section{Symplectic $\mathbb{C} P^{2}$, SympleCtic Ruled MANifolds, AND THEIR SYMPLECTIC SUBMANIFOLDS}

Lemma 6. Let $f:\left(M, \omega_{1}\right) \rightarrow\left(M, \omega_{2}\right)$ be a diffeomorphism such that $f^{*} \omega_{2}=\omega_{1}$. If $A \subset\left(M, \omega_{1}\right)$ is a symplectic submanifold, then $f(A) \subset\left(M, \omega_{2}\right)$ is a symplectic submanifold.

Proof. $f_{*}(T A)=T(f(A))$ is an isomorphism. Take $X, Y \in T A$. Then $\omega_{2}\left(f_{*} X, f_{*} Y\right)$ $=f^{*} \omega_{2}(X, Y)=\omega_{1}(X, Y)$.

$\mathbb{C} P^{2}$ with its standard complex structure carries Kähler forms. Each complex submanifold of $\mathbb{C} P^{2}$ is symplectic with respect to these Kähler forms.

For the symplectic structures on $\mathbb{C} P^{2}$, we have

Theorem $3([\mathrm{~T}])$. If $(M, \omega)$ is a symplectic manifold and $M$ is smoothly diffeomorphic to $\mathbb{C} P^{2}$, then $\omega$ is diffeomorphic to a standard Kähler form.

Lemma 7. Let $u \in H^{2}\left(\mathbb{C} P^{2}\right)$ be a generator. Then a cohomology class $a=l u$ contains a symplectic form of $\mathbb{C} P^{2}$ if and only if $l \neq 0$.

Proof. Any non-zero multiple of the standard symplectic form on $\mathbb{C} P^{2}$ is symplectic.

Lemma 8. For $\left(\mathbb{C} P^{2}, \omega\right)$, let $u \in H^{2}\left(\mathbb{C} P^{2}, \mathbb{Z}\right)$ be a generator. Then any class $\eta=$ au with a being a positive integer can be represented by a connected symplectic submanifold.

Proof. By Theorem 3, $\omega$ is diffeomorphic to a stardard Kähler form. Since $\eta=$ au with $a$ being a positive integer represents a connected complex curve for the standard complex structure, it represents a connected symplectic submanifold for the standard Kähler form. The lemma follows from Lemma 6 ,

Definition 3. An $S^{2}$ fibration over a Riemann surface $\pi: E \rightarrow \Sigma$ is called a ruled manifold.

Ruled manifolds are symplectic manifolds. One can choose the fibration such that the symplectic forms are non-degenerate on the fibers.

Definition 4. A symplectic form on a ruled manifold is compatible with the ruling $\pi: E \rightarrow \Sigma$ if it is non-degenerate on the fibers. 
The following fact is well known.

Lemma 9. Let $E$ be a ruled manifold, and let $F$ be the fiber of $E$. Any cohomology class $a \in H^{2}(E)$ which satisfies the following conditions carries a symplectic form which is non-degenerate on the fibers:

(a) $a(F)$ and $a^{2}(E)$ are positive, and

(b) $a^{2}(E)>(a(F))^{2}$ if the bundle is non-trivial.

With this symplectic form and a compatible complex structure, every connected complex submanifold is a connected symplectic submanifold.

By D. McDuff, we have

Lemma $10(\sqrt{\mathrm{Mc} 2})$. Let $E$ be a ruled manifold, and let $F$ be the fiber of $E$. Then the cohomology class $a \in H^{2}(E)$ of any symplectic form on $E$ which is compatible with the ruling satisfies the conditions

(a) $a(F)$ and $a^{2}(E)$ are positive, and

(b) $a^{2}(E)>(a(F))^{2}$ if the bundle is non-trivial.

For the symplectic forms in the same cohomology class on a ruled manifold, we have

Theorem $4(\overline{\mathrm{LMc}})$. Let $\omega_{0}, \omega_{1}$ be two cohomologous symplectic forms on the ruled 4-manifold $\pi: E \rightarrow \Sigma$. Then there is a diffeomorphism $\Phi$ of $E$ such that $\Phi^{*}\left(\omega_{0}\right)=\omega_{1}$. Moreover, if we assume that the forms $\omega_{0}, \omega_{1}$ are both compatible with $\pi$, then they are isotopic.

We have the following lemmas about the connected symplectic submanifolds of ruled surfaces.

Lemma 11. For $\left(\Sigma_{g} \times S^{2}, \omega\right)$, where $\omega$ is compatible with the ruling, let $x, y$ be the dual class of the fiber $S^{2}$ and the dual class of the base $\Sigma_{g}$, respectively. Then $x$, $y$, and any class $a x+b y$ with $a, b$ being positive integers, can be represented by a connected symplectic submanifold.

If $\int[\omega] \cdot y>n \int[\omega] \cdot x(n=1,2, \ldots)$, then $y-n x$ can be represented by a connected symplectic submanifold.

Lemma 12. For the non-trivial $S^{2}$ bundle $\left(E_{\Sigma_{g}}, \omega\right)$ over $\Sigma_{g}$, where $\omega$ is compatible with the ruling, let $x, y$ be the dual class of the fiber $S^{2}$ and the dual class of the section with self-intersection -1 , respectively. Then $x, y, x+y$, and any class $a x+b y$ with integers $a>1, b>0$ can be represented by a connected symplectic submanifold.

If $\int[\omega] \cdot y>n \int[\omega] \cdot x(n=1,2, \ldots)$, then $y-n x$ can be represented by a connected symplectic submanifold.

Proof of Lemma 11] The classes $x$ and $y$ clearly represent symplectic submanifolds. For the classes $a x+b y$ with $a, b$ being positive integers, we can construct their symplectic representatives. Take the class $p_{i} \times S^{2}$, where $i=1,2, \ldots, a$, and take the class $\Sigma_{g} \times q_{j}$, where $j=1,2, \ldots, b$. These are symplectic submanifolds and they intersect positively transversely at $a b$ points. Then one can smooth out the intersection points to obtain a smooth submanifold which is symplectic and represented by the class $a x+b y$.

When the symplectic area of the base is $n$ times larger than that of the fiber, the class $y-n x$ represents the section at $\infty$ of the projectivised bundle $P(L \oplus \mathbb{C})$, 
where $L$ is a complex line bundle of degree $2 n$ over $\Sigma_{g}$. So it represents a symplectic submanifold.

Alternatively, one may choose compatible complex structures on ruled manifolds and argue that some classes represent complex submanifolds. The proof of Lemma 12 is similar.

\section{Gluing neighborhoods together}

In Section 2, we constructed "local pieces". We will glue these "local pieces" together along their boundaries. Let $L_{1}$ and $L_{2}$ be two "adjacent" "local pieces" such that $\phi\left|L_{1} \leq \phi\right| L_{2}$. Let $a$ be the regular value such that $\phi^{-1}(a)$ is the level along which we glue $L_{1}$ and $L_{2}$. Suppose $\phi_{-}^{-1}(a)$ is the boundary of $L_{1}, \phi_{+}^{-1}(a)$ is the boundary of $L_{2}$, and we will identify them to get $\phi^{-1}(a)$. Now let $M_{a}^{-}=$ $\phi_{-}^{-1}(a) / S^{1}$, and $M_{a}^{+}=\phi_{+}^{-1}(a) / S^{1}$. Then $\phi_{-}^{-1}(a)$ is a principal circle bundle over $M_{a}^{-}$, and $\phi_{+}^{-1}(a)$ is a principal circle bundle over $M_{a}^{+}$. Let $\omega_{-}$and $\omega_{+}$be the reduced symplectic forms on $M_{a}^{-}$and on $M_{a}^{+}$, respectively. We state the following lemma for convenience; it is obviously valid.

Lemma 13. Assume that the two principal circle bundles $\phi_{-}^{-1}(a) \rightarrow M_{a}^{-}$and $\phi_{+}^{-1}(a) \rightarrow M_{a}^{+}$have the same Euler class. If there is a symplectomorphism $g$ between $\left(M_{a}^{-}, \omega_{-}\right)$and $\left(M_{a}^{+}, \omega_{+}\right)$, then $g$ induces an isomorphism between $\phi_{-}^{-1}(a)$ and $\phi_{+}^{-1}(a)$.

In our case, of course the two circle bundles have the same Euler class. As we have seen in Section 3, our reduced spaces are diffeomorphic to $\mathbb{C} P^{2}$ or diffeomorphic to a ruled manifold. $M_{a}^{-}$and $M_{a}^{+}$are diffeomorphic as smooth manifolds. The cohomology classes of the reduced symplectic forms $\omega_{-}$on $M_{a}^{-}$and $\omega_{+}$on $M_{a}^{+}$ agree. By Theorem 3 or by Theorem 4 , there is a diffeomorphism $g:\left(M_{a}^{-}, \omega_{-}\right) \rightarrow$ $\left(M_{a}^{+}, \omega_{+}\right)$such that $g^{*} \omega_{+}=\omega_{-}$. By Lemma 13, this symplectomorphism $g$ between the reduced spaces induces an isomorphism between $\phi_{-}^{-1}(a)$ and $\phi_{+}^{-1}(a)$. We call the identified level set $\phi^{-1}(a)$.

\section{Proof of Theorem 2}

We are now ready to construct our manifolds. It will boil down to the question of choosing some parameters suitably.

Proof. In the following, we always use $\alpha_{0}$ to denote the size of the minimum if the minimum is a surface, and use $t_{0}, t_{1}, t_{2}$ to denote the distances between the moment map images of the fixed point set components as the moment map goes from the minimum to the maximum. For convenience, we will use the following pictures. On the left, between the critical sets, we indicate the Euler class of the principal $S^{1}$ bundle over the reduced space at that regular interval, and on the right of the index 2 surface, we indicate the dual class of the index 2 surface in the reduced space.

By Section 5, the gluing of the "local pieces" can always be done; therefore we will not "glue explicitly" in the following proof, we will check requirements $\mathbf{1}$ and 2 listed in Section 3.

For a manifold of type (1), we have seen an example in $[\mathrm{L}]$. Let us use the construction method to prove its existence. We have the following picture $(u \in$ 
$H^{2}\left(\mathbb{C} P^{2}\right)$ is a generator):

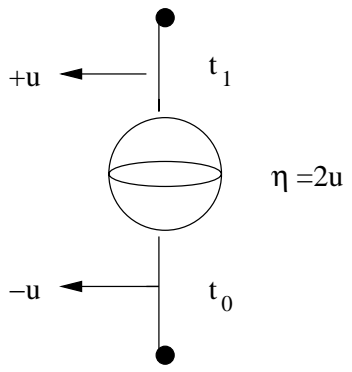

The reduced space at any non-extremal level is $\mathbb{C} P^{2}$.

Since the minimum and the maximum are unique, $t_{0}>0$, and $t_{1}>0$.

Let us use the Duistermaat-Heckman formula ([DH]) to compute the cohomology classes of the reduced symplectic forms. If $0<t \leq t_{0}$, then $\left[\omega_{t}\right]=u t$, by Lemma 7 for each $t$ in this interval, $\left[\omega_{t}\right]$ contains a symplectic form.

By Lemma 8 , the index 2 sphere is a symplectic submanifold of $\mathbb{C} P^{2}$.

Starting new at $t_{0}$, by the Duistermaat-Heckman formula, for $0<t \leq t_{1},\left[\omega_{t}\right]=$ $t_{0} u-u t$. Since $\left[\omega_{t_{1}}\right]=0, t_{1}=t_{0}$. By Lemma 7 again, each $\left[\omega_{t}\right]$ in this interval contains a symplectic form.

This proves the existence of manifold of type (1) in Theorem 1 .

We can similarly prove the existence of manifold of type (2) in Theorem 1 .

For manifold(s) of type (3), we saw in [L] that only when $b_{\text {min }}=1$ can $\phi$ cross the index 4 point before it crosses the index 2 sphere. In this case, we constructed a toric variety. So let us assume the moment map crosses the critical sets in the following order:

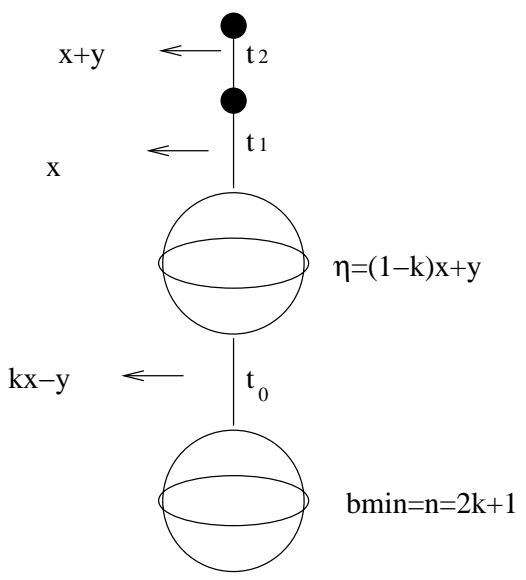

Since the minimum and the maximum are unique, $t_{0}>0, t_{2}>0$.

Note that up to the index 4 critical level (excluding this critical level and the minimal level), the reduced space is $\mathbb{C} \tilde{P}^{2}\left(\mathbb{C} P^{2}\right.$ blown up at a point). By the Duistermaat-Heckman formula, if $0<t \leq t_{0}$, then $\left[\omega_{t}\right]=\alpha_{0} x-(k x-y) t=$ $\left(\alpha_{0}-k t\right) x+t y$. To make $\left[\omega_{t}\right]$ contain symplectic forms for each $t$, by Lemma 9 we can choose $\alpha_{0}>(1+k) t$ for each $t$ in this interval. Since $\alpha_{0}>0$, we only need $\alpha_{0}>(1+k) t_{0}$. 
By Lemma 12 if $k \leq 1$, then $\eta$ represents a connected symplectic submanifold (this imposes no condition); if $k>1$, then if $\alpha_{0}-2 k t_{0}>0, \eta$ represents a connected symplectic submanifold.

For the next interval, let us start with $t=0$. So when $0 \leq t<t_{1}$ (the reduced space $M_{\text {red }}$ at the index 4 critical level is $\mathbb{C} P^{2}$ ),

$$
\left[\omega_{t}\right]=\left[\omega_{t_{0}}\right]-x t=\left(\alpha_{0}-k t_{0}-t\right) x+t_{0} y .
$$

To make $\left[\omega_{t}\right]$ contain a symplectic form for each $t$, we choose $\alpha_{0}-(k+1) t_{0}>t$ for $0 \leq t<t_{1}$.

After $\phi$ crosses the index 4 point, $M_{\text {red }}$ is $\mathbb{C} P^{2}$ and $x+y$ is a generator of $H^{2}\left(\mathbb{C} P^{2}\right)$. If we do the computation starting from the top, we see that each cohomology class in this interval contains a symplectic form. To make the data compatible, we need

$$
\left(\alpha_{0}-k t_{0}-t_{1}\right) x+t_{0} y=t_{2}(x+y),
$$

i.e., $t_{2}=t_{0}$, and $\alpha_{0}=t_{1}+(k+1) t_{0}$.

In summary, we choose the parameters such that

$$
\alpha_{0}>2 k t_{0}, \quad \alpha_{0}=t_{1}+(k+1) t_{0}, \quad t_{1}>0 \quad\left(t_{0}=t_{2}\right) .
$$

For each fixed $k$, we can adjust $\alpha_{0}, t_{0}, t_{1}$ such that the above condition is satisfied. This proves the existence of a manifold(s) of type (3).

We have shown in $\mathrm{L}$ that a manifold of type (4) is diffeomorphic to $\mathbb{C} P^{3}$.

For a manifold of type (5), let us prove the existence for the case when $\phi$ crosses the index 4 point before it crosses the index 2 point:

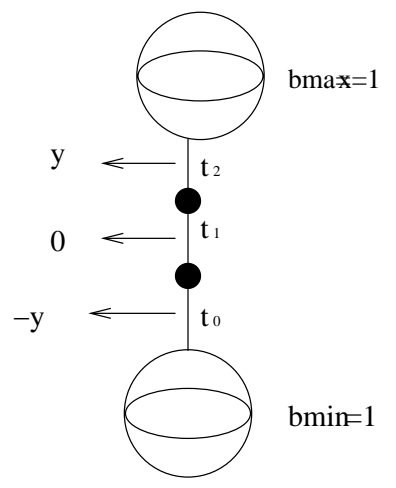

If $0<t<t_{0}, M_{\text {red }}=\mathbb{C} \tilde{P}^{2}$, then $\left[\omega_{t}\right]=\alpha_{0} x+t y$, by Lemma 9 we may choose $\alpha_{0}>t$ for each $t$ in this interval to make $\left[\omega_{t}\right]$ contain symplectic forms. Since $M_{\text {red }}$ at the index 4 point critical level is $\mathbb{C} P^{2}\left(x+y \in H^{2}\left(\mathbb{C} P^{2}\right)\right.$ is a generator $)$, to make the cohomology class contain a symplectic form for $t=t_{0}$, by Lemma 7 we choose $\alpha_{0}=t_{0}$.

Notice that the Euler class of the principal $S^{1}$ bundle over $M_{\text {red }}$ for the $t_{1}$ interval is 0 . We can do a similar computation starting from the top and choose $\tilde{\alpha}=t_{2}$, where $\tilde{\alpha}$ is the size of the maximum. To make the data compatible (the cohomology class of the reduced symplectic form at the index 4 critical level and at the index 2 critical level should be the same) we should choose $t_{0}=t_{2}$.

In summary, we can choose parameters such that

$$
\alpha_{0}=\tilde{\alpha}=t_{0}=t_{2} .
$$


This proves the existence of a manifold of type (5).

For manifolds of type (6a), we will prove the existence for the case when $b_{\min }=$ $n=2 k$ is even (the reduced space is the trivial $S^{2}$ bundle over $\Sigma_{g}$ at any nonextremal level). The proof for the case when $b_{\min }$ is odd is similar (the reduced space is the non-trivial $S^{2}$ bundle over $\Sigma_{g}$ at any non-extremal level).

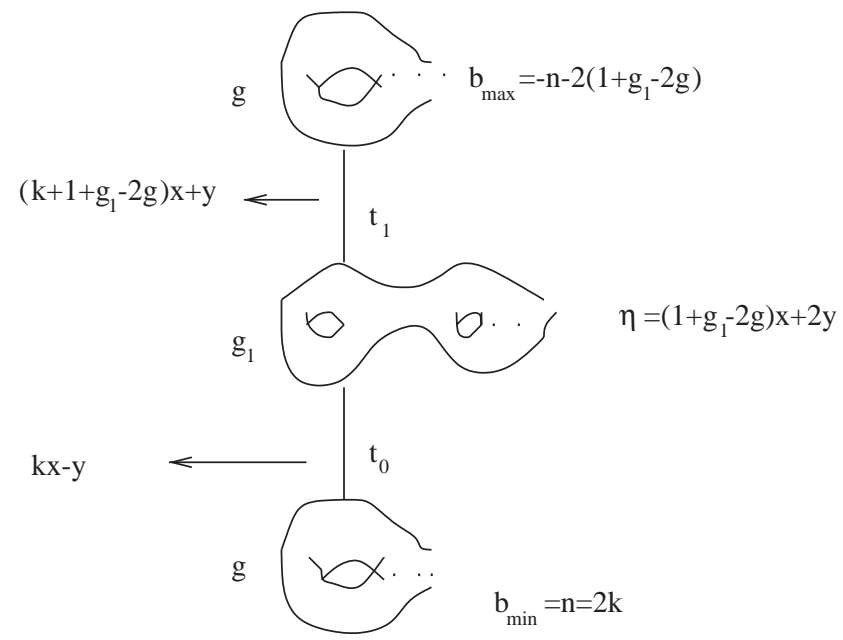

For $0<t \leq t_{0},\left[\omega_{t}\right]=\alpha_{0} x-(k x-y) t=\left(\alpha_{0}-k t\right) x+t y$. By Lemma 9 , we need $\alpha_{0}-k t>0$ for each $t$ to make each cohomology class contain a symplectic form. Since $\alpha_{0}>0$, we only need $\alpha_{0}>k t_{0}$.

By Lemma [1] when $1+g_{1}-2 g>0$, the index 2 surface is a symplectic submanifold of the reduced space.

For $0<t \leq t_{1}$,

$$
\begin{aligned}
{\left[\omega_{t}\right] } & =\left(\alpha_{0}-k t_{0}\right) x+t_{0} y-\left[\left(k+1+g_{1}-2 g\right) x+y\right] t \\
& =\left[\alpha_{0}-k t_{0}-\left(k+1+g_{1}-2 g\right) t\right] x+\left(t_{0}-t\right) y .
\end{aligned}
$$

We need $t_{0}=t_{1}$, and $\alpha_{0}-k t_{0}-\left(k+1+g_{1}-2 g\right) t_{1}>0$, i.e., $\alpha_{0}-n t_{0}>\left(1+g_{1}-2 g\right) t_{0}$. If $k \geq 0$, it is easy to see this choice makes each cohomology class in this interval contain a symplectic form. If $k<0$, then we need $\alpha_{0}-k t_{0}-k t>\left(1+g_{1}-2 g\right) t$ for each $0<t<t_{0}$ to make the cohomology classes contain symplectic forms.

In summary, we make the following choices of the parameters:

$$
\alpha_{0}-k t_{0}-k t>\left(1+g_{1}-2 g\right) t>0, t \in\left[0, t_{0}\right]
$$

We can adjust $\alpha_{0}$ and $t_{0}$ such that the above condition is satisfied for any fixed $k$ and $g, g_{1}$ such that $1+g_{1}-2 g>0$. Therefore manifolds of type (6a) exist when $1+g_{1}-2 g>0$.

For manifolds (6b) (twisted case), $M_{\text {red }}$ is $S^{2} \times S^{2}$. 
When $b_{\min }=n=2 k$ and $b_{\max }=0$, we have the following picture:

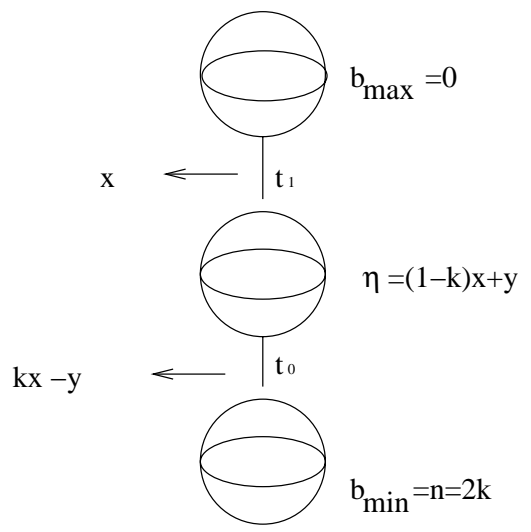

Notice that $t_{0}>0$, and $t_{1}>0$.

When $0<t \leq t_{0},\left[\omega_{t}\right]=\alpha_{0} x-(k x-y) t=\left(\alpha_{0}-k t\right) x+t y$, it is easy to see that when $\alpha_{0}>k t_{0}$, each $\left[\omega_{t}\right]$ contains a symplectic form.

By Lemma 11, if $k \leq 1$, then $\eta$ can be represented by a symplectic submanifold; if $k>1$, then when $\alpha_{0}-(n-1) t_{0}>0, \eta$ can be represented by a symplectic submanifold.

When $0 \leq t \leq t_{1}$ (new start at $\left.t=0\right),\left[\omega_{t}\right]=\left(\alpha_{0}-k t_{0}\right) x+t_{0} y-x t=$ $\left(\alpha_{0}-k t_{0}-t\right) x+t_{0} y$. Since there is a twist, when $t=t_{1}$, we should have

$$
\alpha_{0}-k t_{0}-t_{1}=0 \text {. }
$$

Notice that this kind of choice makes $\left[\omega_{t}\right]$ contain a symplectic form for each $0<$ $t<t_{1}$.

In summary, we can choose

$$
\alpha_{0}>(n-1) t_{0}, \quad t_{1}=\alpha_{0}-k t_{0}>0 .
$$

For each fixed $k$, the choice is achievable. This proves the existence of a manifold of type (6b).

If this manifold exists, then its upside down also exists.

\section{ACKNOWLEDGEMENT}

I thank Denis Auroux for explaining to me the symplectic submanifolds of ruled surfaces. I also thank him for explaining to me the Hurwitz formula for complex maps between Riemann surfaces. For type (6a), the idea of the proof of the nonexistence of the symplectic submanifolds in ruled surfaces for the cases $1+g_{1}-2 g \leq 0$ is due to him.

I thank the referee for his invaluable comments and constructive suggestions.

\section{REFERENCES}

[A] M. Atiyah, Convexity and commuting Hamiltonians, Bull. Lond. Math. Soc. 14 (1982), 1-15. MR.0642416 (83e:53037)

[DH] J. J. Duistermaat and G. J. Heckman, On the variation in the cohomology of the symplectic form of the reduced phase space and Addendum, Invent. Math. 69, 259-269 (1982); 72, 153-158 (1983). MR0674406 (84h:58051a); MR0696693 (84h:58051b)

[GS] V. Guillemin and S. Sternberg, Birational equivalence in the symplectic category, Invent. Math. 97, 485-522 (1989). MR1005004 (90f:58060) 
[HY] Peichu Hu and Mingze Yang, A theorem of differential mappings of Riemann surfaces, Internat. J. Math. Math. Sci. 17, No. 1 (1994), 189-192. MR1255241 (94k:30103)

[L] H. Li, Semi-free Hamiltonian circle actions on 6-dimensional symplectic manifolds, Trans. Amer. Math. Soc. 355, No. 11, 4543-4568, 2003. MR.1990761 (2004e:53127)

[LMc] F. Lalonde and D. McDuff, The classification of ruled symplectic 4-manifolds, Mathematical Research Letters 3, 769-778 (1996). MR1426534 (98b:57040)

[Mc1] D. McDuff, The moment map for circle actions on symplectic manifolds, Journal of Geometry and Physics 5, 149 (1988). MR1029424 (91c:58042)

[Mc2] D. McDuff, The structure of rational and ruled symplectic 4-manifolds, J. Amer. Math. Soc. 3 (1990), 679-712. MR1049697 (91k:58042)

[T] C. Taubes, Seiberg-Witten and Gromov invariants, Geometry and physics (Aarhus, 1995), 591-601, Lecture Notes in Pure and Appl. Math., 184, Dekker, New York, 1997. MR.1423194 (97j:57051)

Department of Mathematics, University of Illinois Urbana-Champaign, Urbana, IlliNOIS 61801

E-mail address: hli@math.uiuc.edu

Current address: Department of Mathematics, Instituto Superior Tecnico, Av. Rovisco Pais, 1049-001, Lisbon, Portugal

E-mail address: hli@math.ist.utl.pt 